\title{
Tuberculosis cerebral sin meningitis en un niño inmunocompetente
}

\author{
Mónica Ramírez, Eduardo Cortés, José Betancur y Carlos Garcés
}

\section{Cerebral tuberculosis without meningitis in a immunocompetent child}

Cerebral tuberculosis TB (tuberculomas) without meningitis is an uncommon disease with a high morbidity and mortality. We report on a case that illustrates the complexity of this clinical presentation. An 11 month old, previously healthy male infant was brought to the clinic due to fever present during the last 1.5 months, associated with loss of neurodevelopmental goals and signs of endocranial hypertension. CT scan of the skull revealed dilatation of the ventricular system with transependimary edema; MRI showed multiple intra- and extra-axial micronodular images and hydrocephalus. Studies of CSF (cyto-chemical analysis, staining, culture for aerobes, fungi, mycobacteria, and molecular tests for TB were negative). Empirical management for subacute meningoencephalitis was prescribed complemented with tetraconjugated treatment for TB and steroids. As there was no microbiological isolation, biopsy of a cerebellar lesion was performed, which revealed chronic necrotizing granulomatous inflammation and acid-alcohol resistant bacilli. The diagnosis of cerebral TB without meningeal involvement was confirmed. The objective of the present report is to emphasize the importance of considering this presentation of TB in children, to remark the need of exhaustive search for the etiologic agent by obtaining samples of the different fluids and tissues even if it implies recurring to invasive methods.

Key words: Cerebral tuberculosis; central nervous system tuberculosis; tuberculomas; tuberculous meningitis; children.

Palabras clave: Tuberculosis cerebral; tuberculosis del sistema nervioso central; tuberculoma; meningitis tuberculosa; niños.

\section{Introducción}



a tuberculosis (TBC) es una enfermedad de distribución mundial. Los niños bajo los cinco años representan un grupo etario vulnerable por la alta incidencia de TBC diseminada y del sistema nervioso central (SNC), ambas con rápida progresión desde la infección hacia la enfermedad. Según una publicación de la OMS, en el año 2015 se presentaron un millón de casos nuevos de TBC en niños, que corresponden a $10 \%$ del total de casos notificados en el año ${ }^{1}$. En América en el año 2013, se notificaron un total de 11.379 casos, equivalentes a 4,9 por 100.000 niños ${ }^{2}$; en Colombia en 2016 se informó un total de 13.467 casos, pero sin datos de población infantil ${ }^{3}$.

El compromiso del SNC representa 5 a $10 \%$ de los casos de localización extrapulmonar y la meningitis es el cuadro más frecuente; una forma infrecuente de presentación son el (los) tuberculoma(s) cerebral(es). La TBC del SNC es considerada la manifestación más devastadora de la enfermedad ${ }^{4,5}$, con una mortalidad de hasta $30 \%$ y un alto porcentaje de secuelas neurológicas en los sobrevivientes ${ }^{5}$.

Presentamos el caso clínico de un lactante menor de un año, presuntamente inmunocompetente, con un síndrome febril prolongado, compromiso neurológico e hidrocefalia con una TBC cerebral, sin meningitis, con la finalidad de alertar a la comunidad médica sobre la importancia del diagnóstico.

\section{Caso clínico}

Niño de 11 meses de edad, residente en Medellín, producto de una segunda gestación, parto de término sin complicaciones, previamente sano, neurodesarrollo normal y esquema de vacunación completo para la edad incluyendo BCG. Presentó un cuadro clínico de un mes y medio de evolución de un síndrome febril intermitente, disminución del apetito, decaimiento y pérdida de logros en el neurodesarrollo (dejó de gatear, perdió el tono axial y se tornó irritable). Consultó en varias ocasiones en que se le indicaron varios tratamientos que incluyeron amoxicilina/ácido clavulánico, medicamentos homeopáticos y analgésicos comunes, sin mejoría clínica. Dos días antes del ingreso presentó un empeoramiento de los síntomas con mayor irritabilidad, supraversión de la mirada, posiciones tónicas y tendencia al opistótonos.
Clínica Las Américas, Medellín, Colombia.

Sin conflictos de interés. Sin fuente de financiamiento.

Recibido: 10 de mayo de 2017 Aceptado: 13 de marzo de 2018

Correspondencia a: Mónica M. Ramírez monicaram@une.net.co 

tórax portátil al ingreso. Se observa compromiso intersticial micronodular pulmonar bilateral compatible con TBC miliar.
Figura 1. Radiografía de

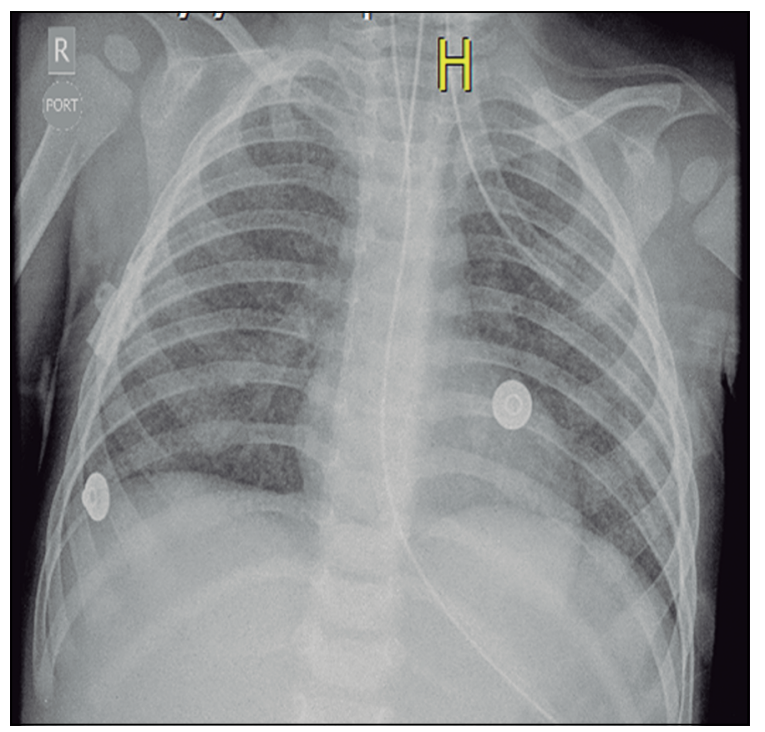

\section{RESONANCIA AL INGRESO: 21/05/2015}



Figura 2. Resonancia magnética de cráneo con contraste al ingreso. Se observan múltiples lesiones nodulares inespecíficas infra y supratentoriales que realzan con el contraste, asociadas a hidrocefalia obstructiva.
En el centro de consulta inicial se realizó el diagnóstico de una posible meningitis; la TC de cráneo mostró una dilatación del tercer ventrículo y laterales. En esa oportunidad no se realizó punción lumbar; se inició tratamiento empírico con vancomicina, ceftriaxona y dexametasona, siendo remitido al día siguiente a nuestra institución.

No tenía antecedentes de importancia ni contacto identificado con personas con síntomas respiratorios o con TBC.

Al ingreso, se encontraba en malas condiciones generales, afebril, presión arterial 138/96 (hipertensión estadio 2 para la edad/talla), frecuencia cardíaca 108 por min y respiratoria 24 por min, estado de conciencia fluctuante entre irritabilidad y somnolencia, con episodios intermitentes de hipertonía generalizada con posición de opistótonos, pupilas isocóricas y reactivas, hemiparesia derecha, movimientos atáxicos, dismetría y reflejos osteotendinosos exaltados en extremidades inferiores. Presentaba signos clínicos de hipertensión intracraneana dados por hipertensión arterial, bradicardia (hasta 70/min) y frecuencia respiratoria bajas para la edad, fondo de ojo con papiledema grado I-II. El resto del examen físico: no tenía adenopatías, auscultación cardiopulmonar normal y abdomen sin masas o visceromegalias.

El TC simple de cráneo inicial mostró una ventriculomegalia con edema transependimario triventricular, infiltración difusa del mesencéfalo y la protuberancia que colapsaba parcialmente el cuarto ventrículo.

Se obtuvo LCR por punción ventricular. El estudio citoquímico fue normal ( $\mathrm{pH} \mathrm{7,5;} \mathrm{glucosa} 91 \mathrm{mg} / \mathrm{dL}$, eritrocitos 78 y leucocitos 1 cél $/ \mathrm{mL}$, proteínas $22,5 \mathrm{mg}$ / $\mathrm{dL}$, antígenos bacterianos negativos por látex; tinción y cultivo para bacterias aerobias, hongos y micobacterias negativos; pruebas moleculares (RPC en tiempo real) para Mycobacterium tuberculosis, herpes simplex tipo 1 y 2, y enterovirus negativos; y citología negativa para malignidad. El estudio de contenido gástrico (tres muestras) para $M$. tuberculosis (Ziehl-Neelsen y cultivo) fueron negativos. La serología para VIH, TORCH (toxoplasma, herpes, citomegalovirus y rubéola) y la prueba de tuberculina también resultaron negativos.

La radiografía de tórax del ingreso mostró un compromiso intersticial micronodular bilateral compatible con una TBC miliar (Figura 1). La resonancia magnética (RM) cerebral reveló múltiples lesiones nodulares inespecíficas infra y supratentoriales que realzaban con el contraste, asociadas a una hidrocefalia obstructiva (Figura 2).

El estado clínico del paciente empeoró en las siguientes $36 \mathrm{~h}$ del ingreso, con compromiso de conciencia y signos de hipertensión endocraneana. Ante el diagnóstico de una meningoencefalitis subaguda y romboencefalitis sin etiología se inició tratamiento antimicrobiano empírico con vancomicina $60 \mathrm{mg} / \mathrm{kg} /$ día, ceftriaxona $100 \mathrm{mg} / \mathrm{kg} /$ día 
y aciclovir $1.500 \mathrm{mg} / \mathrm{m}^{2}$ al día y terapia antituberculosa tetraconjugada con isoniazida $10 \mathrm{mg} / \mathrm{kg} / \mathrm{día}$, rifampicina $10 \mathrm{mg} / \mathrm{kg} /$ día, etambutol $15 \mathrm{mg} / \mathrm{kg} /$ día y pirazinamida $25 \mathrm{mg} / \mathrm{kg} /$ día. Además, recibió terapia corticoesteroidal (dexametasona $0,6 \mathrm{mg} / \mathrm{kg} /$ día); anticonvulsivantes y antiedema cerebral con solución salina hipertónica.

Debido a la falta de diagnóstico microbiológico del LCR se realizó una biopsia de una lesión cerebelar por craniotomía posterior cuyo estudio histopatológico mostró una inflamación granulomatosa crónica necrosante, con tinción de plata metenamina negativa y de Ziehl-Neelsen positiva para bacilos ácido-alcohol resistentes (BAAR) (Figura 3). Así, al confirmarse el diagnóstico de TBC cerebral se continuó la terapia antituberculosa y los corticoesteroides por cuatro semanas, completando el paciente un mes de internación.

Un mes después del alta, el paciente se encontraba recuperando los hitos del desarrollo perdidos, comen-



Figura 3. Anatomía patológica de la muestra de biopsia de cerebelo. A. Tinción de Ziehl Neelsen 100X. Bacilo ácido alcohol resistente en tejido cerebelar (flecha roja). B. Tinción de hematoxilina/eosina 40X. Inflamación granulomatosa crónica con necrosis de caseificación central (flecha roja) y célula gigante multinucleada de tipo Langhans (flecha azul) en medio del granuloma. zando a caminar con ayuda y sin convulsiones $u$ otras complicaciones.

En el estudio de los contactos, la madre tuvo baciloscopias positivas, a pesar de no acusar síntomas respiratorios. No tenía antecedentes de consumo de tabaco ni contactos conocidos con $\mathrm{TBC}$, por lo que recibió tratamiento antituberculoso acortado.

A los tres meses del alta, el paciente estaba en mejores condiciones clínicas, logrando deambulación sin apoyo e iniciando el lenguaje con monosílabos. El estudio de potenciales auditivos de tallo cerebral y la audiometría fueron normales. La RM cerebral de control a los tres meses (Figura 4) mostró cerebelomalacia en el área de la biopsia; pequeños nódulos múltiples a nivel supratentorial, regiones interhemisférica, temporal y parietal

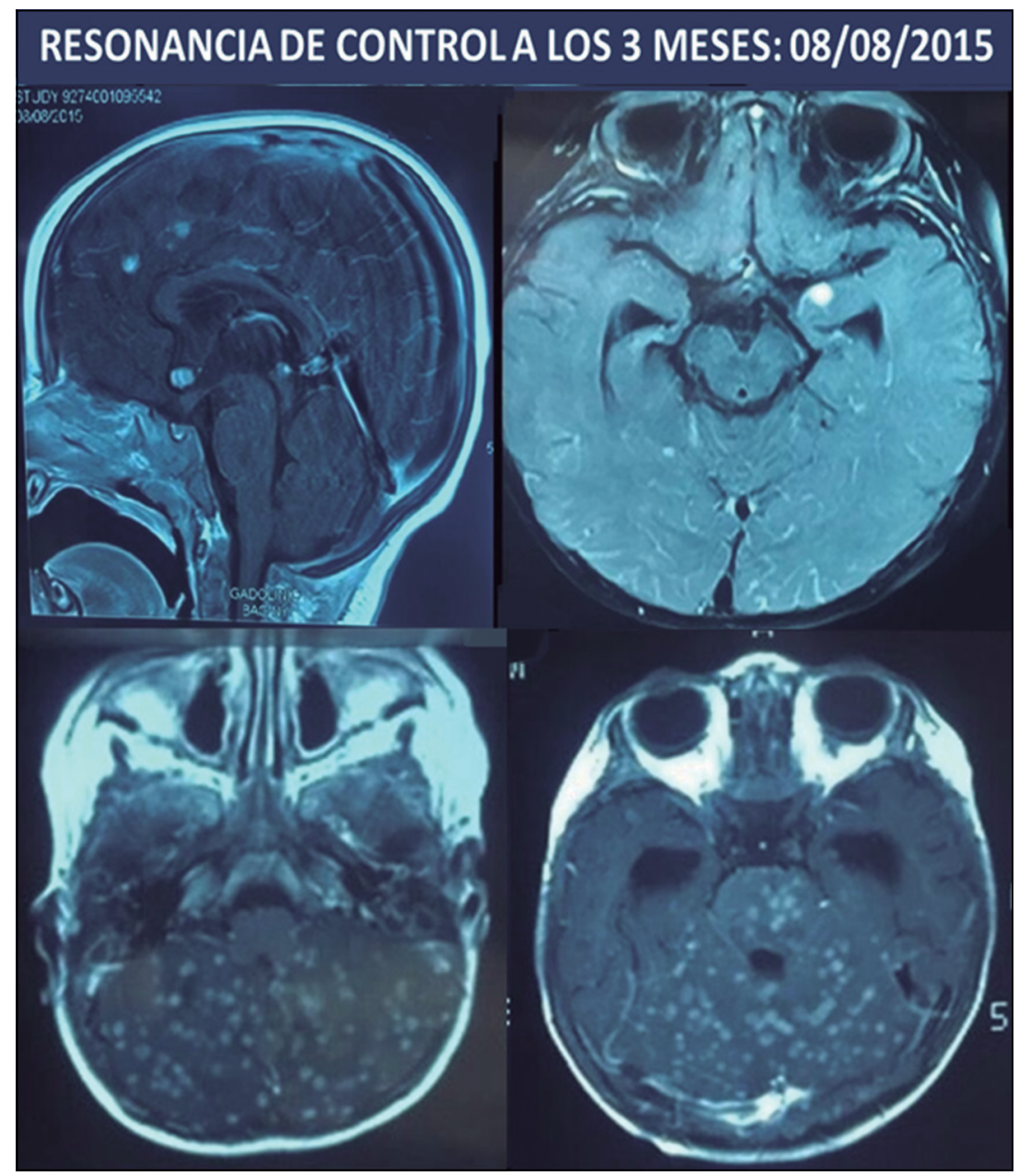

Figura 4. Resonancia magnética a los tres meses del diagnóstico. Se aprecian los siguientes cambios: Pequeña área de cerebelomalacia en el sitio de la biopsia; pequeños nódulos múltiples supratentoriales, y en regiones interhemisférica, opérculo frontal derecho, temporal y parietal, compatibles con tuberculomas no caseificantes; y ausencia de realces anormales infratentoriales, aracnoiditis, vasculitis o hidrocefalia. Estos cambios reflejaban mejoría con respecto al estudio inicial, sobre todo en la hidrocefalia. 
compatibles con tuberculomas no caseificantes; $y$ ausencia de realces anormales en la región infratentorial, así como de signos de aracnoiditis, vasculitis, o hidrocefalia (Figura 4). Estos cambios reflejaban mejoría evidente con respecto al estudio inicial.

En el control a los 15 meses del diagnóstico, se encontraba con evolución clínica favorable, con neurodesarrollo normal para la edad y sin secuelas evidentes al examen físico. En la evaluación imagenológica, la RM presentaba una pequeña área de encefalomalacia del giro frontal superior derecho y no se observaban lesiones hipercaptantes en el parénquima cerebral, ni evidencia de hidrocefalia (Figura 5). Como estudio complementario se realizó una evaluación por inmunología practicándose secuencia del exoma a la madre y al paciente que resultó normal, sin ninguna alteración genómica que hubiera explicado la susceptibilidad a infecciones tipo TBC.

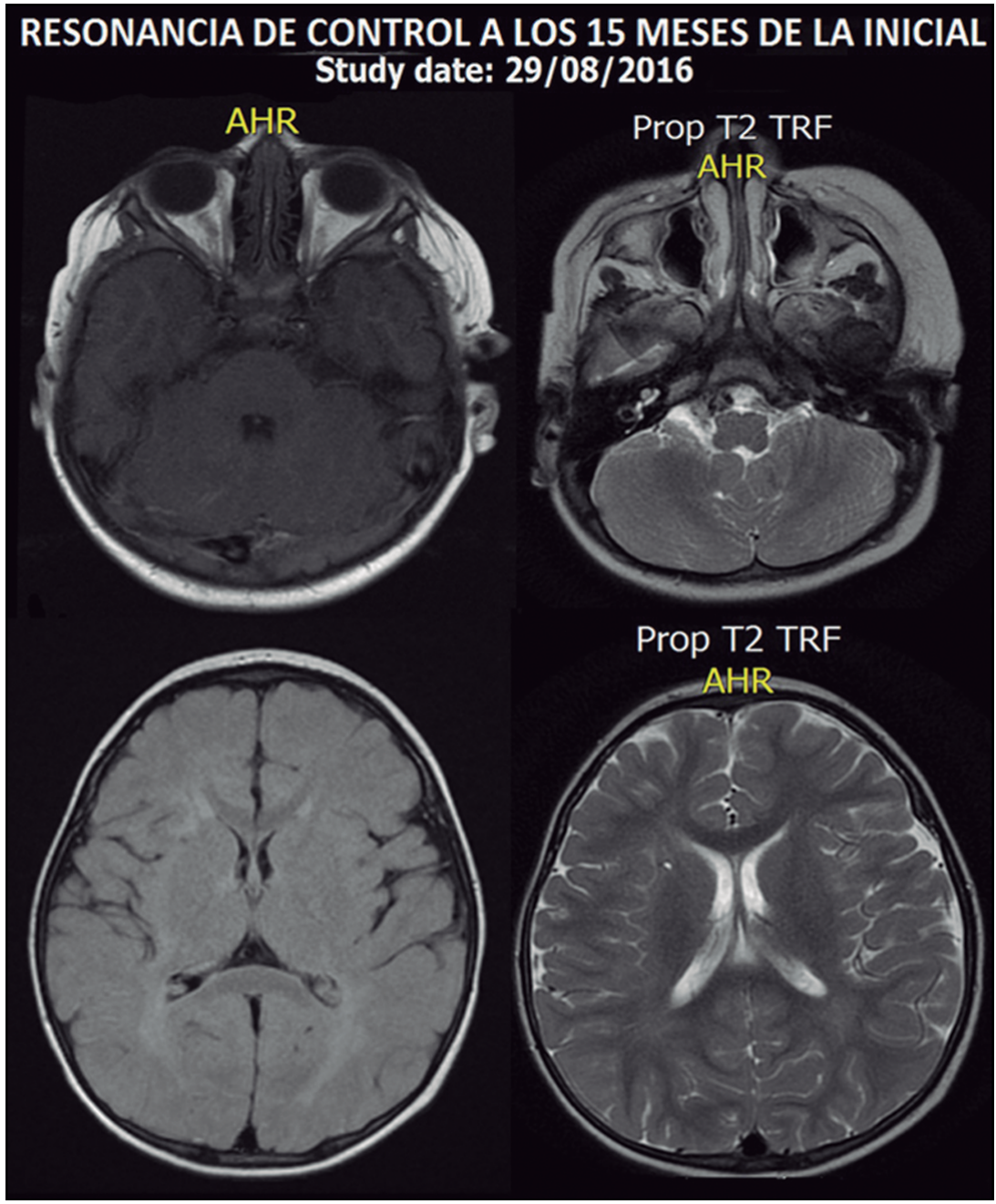

Figura 5. Resonancia magnética cerebral de control a los 15 meses de la inicial. No se observan lesiones hipercaptantes en el parénquima cerebral, ni hay evidencia de hidrocefalia.

\section{Discusión}

La infección ocurre generalmente por inhalación de aerosoles que contienen el bacilo y éste se deposita en los alvéolos. El bacilo se filtra a los ganglios linfáticos, pudiendo ocurrir una bacteriemia con diseminación a cualquier parte del organismo. El compromiso del SNC empieza con un foco tuberculoso vascular caseificante en cerebro, médula espinal o meninges; la meningitis tuberculosa se presenta cuando este foco se rompe al espacio subaracnoideo. Este fenómeno desencadena una respuesta inflamatoria con exudado denso, que puede obstruir el flujo del líquido cefalorraquídeo (LCR), causando hidrocefalia obstructiva en el 70\% de los casos y compresión de los nervios craneales. Los tuberculomas se presentan cuando $M$. tuberculosis alcanza el parénquima cerebral por vía hematógena, dando lugar a los focos de Rich que permanecen controlados por la respuesta inmunológica o pueden evolucionar a tuberculomas; otra forma de presentación son los infartos cerebrales por vasculitis que afectan principalmente vasos sanguíneos en la base del cerebro ${ }^{4,6}$.

La TBC del SNC puede presentarse de tres diferentes formas: meningitis tuberculosa, tuberculoma (meníngeo o cerebral) y absceso tuberculoso ${ }^{4,7,8}$.

La meningitis por TBC generalmente se manifiesta como una enfermedad febril subaguda con tres fases o estadios: el estadio I con inicio insidioso de malestar general, fiebre de bajo grado y cambios de comportamiento durante dos a tres semanas; estadio II con signos meníngeos y alteración del estado de conciencia, puede haber compromiso de pares craneales e hidrocefalia; y estadio III que progresa rápidamente con convulsiones y coma, de alta mortalidad. La mayoría de los casos se diagnostica en la segunda fase ${ }^{9}$.

El tuberculoma de SNC se caracteriza histológicamente por una zona central de necrosis caseosa rodeada por una cápsula de tejido fibroso, células epitelioides, células gigantes multinucleadas y linfocitos; en el centro necrótico hay BAAR viables en estado latente ${ }^{10}$. Los tuberculomas pueden encontrarse en cerebro, cerebelo, espacio subaracnoideo, subdural, epidural y médula espinal; generalmente la localización es infratentorial en niños, mientras que en adultos las lesiones tienden a ser supratentoriales y a menudo se ubican en la unión corticomedular o en regiones periventriculares ${ }^{11}$. Wasay y cols. reportaron en 100 pacientes, un número variable entre una y más de 100 lesiones, con un promedio de cuatro por paciente. Un tercio de ellos se presentaron como una lesión única ${ }^{11}$.

Los abscesos tuberculosos son una manifestación infrecuente del compromiso neurológico. Se presentan como una lesión purulenta que contiene bacilos viables sin evidencia del granuloma caseificante típico. Las 
manifestaciones clínicas dependen de la localización y frecuentemente cursan con signos de hipertensión endocraneana y déficit neurológico focal ${ }^{4}$.

La hidrocefalia se presenta en $80 \%$ de los casos de TBC meníngea ${ }^{6}$ y en la mayoría es de tipo comunicante. La forma obstructiva se debe principalmente al efecto de masa secundario a tuberculomas o abscesos cerebrales ${ }^{6,12,13}$. En nuestro paciente fue de tipo obstructivo y mejoró con tratamiento médico sin necesidad de derivación.

La duración de los síntomas de la TBC del SNC es generalmente de tres semanas antes del ingreso hospitalario; la mayoría se presenta con alteración del estado de conciencia, déficit neurológico focal y fiebre. Los signos meníngeos y las convulsiones también son frecuentes ${ }^{14}$, y en la gran mayoría se hace el diagnóstico en el estadio II $^{8,15}$.

El diagnóstico de TBC del SNC en niños es difícil, considerando que sólo alrededor de 50\% tienen la prueba cutánea de tuberculina positiva y hasta el $60 \%$ tienen radiografía de tórax normal, a diferencia del caso presentado en el que se observó infiltrado micronodular bilateral compatible con TBC miliar ${ }^{6,15,16}$.

El contacto con un caso sospechoso o confirmado de TBC también ayuda al diagnóstico, pero en la mitad de los pacientes no se documenta el caso índice ${ }^{12}$. En nuestro paciente, la detección de BAAR en esputo en la madre, sustenta la indicación de hacer una búsqueda activa del caso índice.

En la meningitis tuberculosa, el análisis del LCR obtenido ya sea por punción ventricular o punción lumbar es prioritario; generalmente hay proteínas elevadas, glucosa baja y pleocitosis a expensas de leucocitos mononucleares. La sensibilidad del estudio del LCR para la detección de TBC mejora con volúmenes grandes o muestras múltiples ${ }^{4,7}$, lo cual no siempre es factible obtener. La tinción para BAAR en LCR es positiva en alrededor de $30 \%$ y el aislamiento en cultivos puede alcanzar $50 \%{ }^{14,15}$. La adenosina desaminasa (ADA) es una enzima producida por linfocitos T que se puede cuantificar en LCR; en 63 a $100 \%$ de pacientes con meningitis tuberculosa se observan cifras elevadas ${ }^{17}$. El estudio molecular en el LCR se ha comenzado a utilizar con una frecuencia creciente; la OMS reporta datos de sensibilidad de 59-84\% y de especificidad entre 73 y $89 \%$ para la prueba Xpert MTB/ RIF recomendada ${ }^{6}$.

En el caso presentado no había alteraciones del LCR obtenido por punción ventricular ni imagenológicas sugerentes de una meningitis por TBC o ruptura de tuberculomas al espacio subaracnoideo. El diagnóstico se estableció únicamente con el estudio histopatológico del cerebelo que evidenció una inflamación granulomatosa crónica y presencia de BAAR.

El diagnóstico de TBC del SNC en nuestro paciente, además fue respaldado por la mejoría clínica e imagenológica que tuvo en respuesta al tratamiento antituberculoso instaurado. El tratamiento de la TBC de SNC debe comprender dos meses de terapia de inducción con esquema tetraconjugado, seguido por 10 meses de mantenimiento con rifampicina e isoniazida como primera línea de manejo ${ }^{5}$.

En cuanto al pronóstico, los principales factores de riesgo descritos en nuestro paciente fueron el estadio III de la enfermedad, coma profundo y la edad bajo los dos años, con secuelas de predominio neurológico y auditivo hasta en el $77 \%{ }^{18}$; en Colombia se ha reportado una mortalidad de $17 \%{ }^{19}$. La vacunación con BCG tiene una protección estimada para TBC meníngea de $73 \%$ y para TBC miliar de $77 \%{ }^{18}$.

\section{Conclusión}

Nuestro caso ilustra la importancia de tener en cuenta la TBC del SNC entre los diagnósticos etiológicos diferenciales de los pacientes pediátricos incluyendo lactantes, quienes se presenten con un cuadro febril prolongado, pérdida de los logros del neurodesarrollo, y clínica de meningitis subaguda o de síndrome de hipertensión endocraneana. Debe enfocarse en la búsqueda activa del agente etiológico por los diferentes medios, recordando que la TBC cerebral se puede presentar con o sin meningitis y que en ocasiones hay que llegar a métodos invasores como la biopsia de las lesiones como en nuestro caso, pues el diagnóstico y tratamiento tempranos influyen significativamente en la morbimortalidad.

Agradecimientos. Los autores agradecen a la Dirección Científica de Clínica Las Américas por su colaboración en la redacción y la revisión crítica del manuscrito. Y al Laboratorio de Patología Las Américas por las fotografías de la anatomía patológica.

\section{Resumen}

La tuberculosis (TBC) cerebral o tuberculoma(s) sin meningitis es una enfermedad poco frecuente y de alta morbimortalidad. Presentamos el caso clínico de un lactante de 11 meses, previamente sano, que consultó por fiebre prolongada y síntomas neurológicos. La RM de encéfalo mostró múltiples imágenes micronodulares e hidrocefalia. El estudio de LCR para bacterias, hongos y micobacterias fue negativo. Se prescribió terapia empírica como una meningoencefalitis subaguda y tratamiento antituberculoso tetraconjugado y corticoesteroides. La confirmación del diagnóstico de TBC cerebral se realizó por biopsia de la lesión, con presencia de inflamación granulomatosa crónica necrosante y bacilos ácido-alcohol resistentes. Se enfatiza la importancia de considerar esta presentación de TBC en niños, y la necesidad de la búsqueda exhaustiva del agente etiológico en diferentes líquidos y tejidos, aun por métodos invasores. 


\section{Referencias bibliográficas}

1.- OMS | Informe mundial sobre la tuberculosis. WHO. (Fecha de acceso: 19 de enero de 2018). Disponible en: http://www.who.int/tb/ publications/global_report/es/

2.- Pan American Health Organization. World Health Organization Regional Office for the Americas. Tuberculosis in the Americas. Regional Report 2014. Epidemiology, Control and Financing. (Fecha de acceso: 19 de enero de 2018). Disponible en: http://www.paho.org/ hq/index.php?option $=$ com_docman $\& \operatorname{task}=$ doc view\&Itemid $=270 \&$ gid $=31283 \&$ lang $=\mathrm{fr}$

3.- Organización Mundial de la Salud. Tuberculosis: perfiles de países. WHO. (Fecha de acceso: 19 de enero de 2018). Disponible en: http://www.who.int/tb/country/data/profiles/es/

4.- Rock R B, Olin M, Baker C A, Molitor T W, Peterson P K. Central nervous system tuberculosis: pathogenesis and clinical aspects. Clin Microbiol Rev 2008; 21: 243-61. doi: 10.1128/CMR.00042-07.

5.- Jordán Jiménez A, Tagarro García A, Baquero Artigao F, del Castillo Martín F, Borque Andrés C, Romero M P, et al. Meningitis tuberculosa: revisión de 27 años. An Pediatr (Barc) 2005; 62: 215-20. doi: 10.1157/13071835.

6.- $\quad$ van Toorn R, Solomons R. Update on the diagnosis and management of tuberculous meningitis in children. Semin Pediatr
Neurol 2014; 21: 12-8. doi: 10.1016/j. spen.2014.01.006.

7.- $\quad$ Principi N, Esposito S. Diagnosis and therapy of tuberculous meningitis in children. Tuberculosis 2012; 92: 377-83. doi: 10.1016/j. tube.2012.05.011.

8.- Israni A V, Dave D A, Mandal A, Singh A, Sahi P K, Das R R, et al. Tubercular meningitis in children: Clinical, pathological, and radiological profile and factors associated with mortality. J Neurosci Rural Pract 2016; 7 : 400-4.

9.- Ramzan A, Nayil K, Asimi R, Wani A, Makhdoomi R, Jain A. Childhood tubercular meningitis: an institutional experience and analysis of predictors of outcome. Pediatr Neurol 2013; 48: 30-5. doi: 10.1016/j. pediatrneurol.2012.09.004

10.- Ravenscroft A, Schoeman J F, Donald P R. Tuberculous granulomas in childhood tuberculous meningitis: radiological features and course. J Trop Pediatr 2001; 47: 5-12.

11.- Wasay M, Kheleani B A, Moolani M K, Zaheer J, Pui M, Hasan S, et al. Brain CT and MRI findings in 100 consecutive patients with intracranial tuberculoma. J Neuroimaging 2003; 13: 240-7.

12.- Patkar D, Narang J, Yanamandala R, Lawande M, Shah G V. Central nervous system tuberculosis: pathophysiology and imaging findings. Neuroimaging Clin N Am 2012; 22 :
677-705. doi: 10.1016/j.nic.2012.05.006.

13.- Saavedra J S, Urrego S, Pérez Á, Toro M E. Diagnóstico de meningitis tuberculosa. Acta Neurol Colomb 2015; 31: 223-30.

14.- Titone L, Di Carlo P, Romano A, Maggio M C, Salsa L, Abbagnato L, et al. Tuberculosis of the central nervous system in children: 32 years survey. Minerva Pediatr 2004; 56: 611-7.

15.- Farinha N J, Razali K A, Holzel H, Morgan G, Novelli V M. Tuberculosis of the central nervous system in children: a 20-year survey. J Infect 2000; 41: 61-8.

16.- Janse van Rensburg P, Andronikou S, Toorn R van, Pienaar M. Magnetic resonance imaging of miliary tuberculosis of the central nervous system in children with tuberculous meningitis. Pediatr Radiol 2008; 38: 1306-13.

17.- Morales-Aguirre J J. Infección por micobacterias del sistema nervioso central. Bol Med Hosp Infant Mex 2006; 63: 332-50.

18.- González-Saldaña N, Hernández-Porras M, Macías-Parra M, Monroy-Colín V A, AceboArcentales J J, Juárez-Olguin H. Tuberculous meningitis: symptoms, diagnosis and evaluation experienced in 532 patients in a pediatric hospital. Asian Pac J Trop Dis. 2016; 6: 208-11. doi: 10.1016/S2222-1808(15)61015-5.

19.- Henao López A, Vivas Trochez R, Cornejo Ochoa W. Factores pronósticos para secuelas y mortalidad de la tuberculosis meníngea en niños. Acta Neurol Colomb 2011; 27: 211-21. 